\title{
Learning Survival Models with On-Line Simulation Activities in the Actuarial Science Degree
}

\author{
doi:10.3991/ijet.v6i1.1398 \\ A. Fernández-Morales \\ University of Málaga, Málaga, Spain
}

\begin{abstract}
The aim of this paper is to describe an on-line survival laboratory designed to enhance teaching and learning in the Statistics courses of the Actuarial Science Degree of the University of Málaga. The objective of the on-line survival lab is to help students through a guided program of simulation activities with the understanding of the most important statistical concepts of the stochastic modeling of human survival, from an Actuarial point of view. The graphical interactive simulator is implemented as Java applets for the web version, and as a Javascript animation for a lite iPhone/iPod touch version. Finally, the results of a survey carried out at the end of the course are discussed to have a preliminary assessment of the students' satisfaction with the resources, and their perception about the usefulness for their learning process.
\end{abstract}

Index Terms-Applets, interactive learning, simulation, survival models.

\section{INTRODUCTION}

Survival analytical models and related techniques are necessary and essential tools for the actuary's professional activity. However, some students in the first course of the Actuarial Science Degree have difficulties with the understanding of these complex models and show little interest in getting a deeper understanding of them. To face this problem, in an attempt to motivate students and to facilitate their learning process, a series of on-line activities based on an interactive simulator have been integrated into an "on-line survival laboratory" for the students in the first course of the Degree in Actuarial Science at the University of Málaga.

The rationale behind the choice of this type of activities in our courses is that, as some authors stated, [14], using Information Technology (IT) based educational tools for simulation, investigation, or illustration, has the potential to increase both interest in and understanding of the contents. Currently, available IT based educational resources are much more than simple collections of static lecture notes, homework assignments, and tutorials posted on course-specific Internet sites, [7], [13]. Recent approaches provide dynamic, linked, and interactive learning contents, like web-based resources for blended instruction, real-time educational blogs, collaborative learning environments, and resources for monitoring and assessment.

There are a number of examples in the field of Statistics education that combine new pedagogical approaches with technological infrastructure allowing instructors and students to improve motivation and enhance the learning process, [10], [12], [15], or [7]. The most significant experiences in Higher Education in this area integrate interactive resources with IT using interactive learning tools that help with the understanding and assimilation of abstract concepts, which are more difficult to teach and learn in the traditional classroom, [7], [12], [6]. One of the most common tools in this field is the Java applet embedded in an html document, [14], [7], [1], [16]. These applets generally illustrate statistical ideas through simulation, and allow students to investigate relevant ideas, and to construct a deeper understanding of underlying concepts. In addition, as [2] mentions, web-based simulation has some benefits in comparison to classical systems, like easy of use, cross-platform capability, and wide availability, among others.

According to these well-known experiences and previous personal works in the field, [8] and [9], the activities of the on-line survival laboratory are based on a graphical simulator developed as a series of Java applets. In addition, a Java-script based micro simulator for the iPhone has been also introduced as a complementary resource to add more flexibility and variety to the available resources, like other experiences like [4].

Solely providing the technology or the resources is not enough guaranty of better learning; it is necessary to design a cognitive strategy to improve the learning process [5], [6]. To integrate the simulator and the activities into a coherent pedagogical framework, the on-line survival laboratory, which is described in section II, includes a guided program of activities to be carried out using the simulator, and also a section with information of survival models commonly used in the Actuarial Science, links, and references. This structure was designed as an attempt to overcome one of the common disadvantages of Java applets in Statistics education, mentioned by [3], that they are not often accompanied by detailed documentation and activities to guide student use. The main features of the simulator are discussed in section II, too.

The aim of the on-line survival lab is to help students through practice with the understanding of the most important statistical concepts related to human survival and its stochastic modeling, from an Actuarial point of view. In order to have a preliminary assessment of its effectiveness, a short survey has been carried at the end of this academic year. The questionnaire included questions related to the students' satisfaction with the resources, and their perception about the usefulness for their learning process. The results of the survey are discussed in section III. 
Finally, in section IV, the main conclusions of this paper are summarized.

\section{AN ON-LiNE SURVIVAL LABORATORY}

Survival models are the core of the Actuarial Statistics I course in the degree of Actuarial Science at the University of Málaga. Hence, many of the learning activities we designed for our students are based on, or use, analytical survival models. We attempt to enrich these activities with a significant interactive nature. Manipulation and interaction are key aspects of effective learning resources [11], by interaction students construct their own understanding and use of the resource, and simulation plays a significant role in enhancing students' ability to understand and study statistical concepts [3].

We deliver the resources on-line, in order to encourage an autonomous usage. In this way we are also promoting the acquisition of competences related to IT competence and self-learning, which are considered key elements in the new teaching and learning approach following the Bologna process.

However, according to [5] computer simulations are not enough guaranty of better learning; it is necessary to design a cognitive strategy to improve the learning process. Thus, the pedagogical framework that integrates these activities is an on-line laboratory, which includes a guided program of learning based on an interactive simulator of analytical survival models. Once the student gains enough ability using the simulator, he or she can use it by him or herself independently for advanced projects in this and other courses.

\section{A. The Survival Laboratory (SLAB)}

The on-line survival laboratory consists of several linked web pages that contain information about analytical survival models as well as an itinerary of guided activities using the graphical simulator of survival models.

The structure of the on-line lab is summarized in Fig. 1. In order to get a clear structure and easy navigation experience, the components of SLAB are the following:

- An introductory page that explains the aims of this web, the contents of the web laboratory, and its structure.

- Models section. This section consists of five model pages (one per model). In these pages the student can find the main features of each model (De Moivre, Dormoy, Gompertz, Makeham and Heligman-Pollard), along with some historic remarks and links to the pages containing the activities of the selected model. In these pages, the student can also find bibliographical references and more information about survival models.

- Activities section. For each model there are three linked activities pages. Every activity page proposes a problem to be solved using the graphical interactive simulator. There is also a main activities page with direct links to all the activities, classified by model, for the experienced students.

- iPhone/iPod touch section. The last academic year an iPhone specific section has been added to the web laboratory. In this section students can access a lite version of the simulator using their iPhones or iPods, to solve three easy activities, conceived as an introductory tool to engage students in the main on-line version.
The design of all the web pages of the survival on-line lab follows a common structure, in order to simplify the navigation tasks. The links to models pages are located on the left side menu and the upper right side displays direct links to the home page, 'more info' and activities pages.

The activities pages are the central part of the Survival on-line lab. At present, there are 15 activity pages (three for every model) with a structure like the one displayed in fig. 2.

In addition to the common structure of all the web pages of the on-line laboratory, every activity page includes in the upper side the guidelines of the activity: the description of the problem or case to be studied, the specification of the adequate model to be applied, the initial values of the parameters at the launching of the simulator, a concise description of the simulator instructions, and the statistical source if it includes real data.

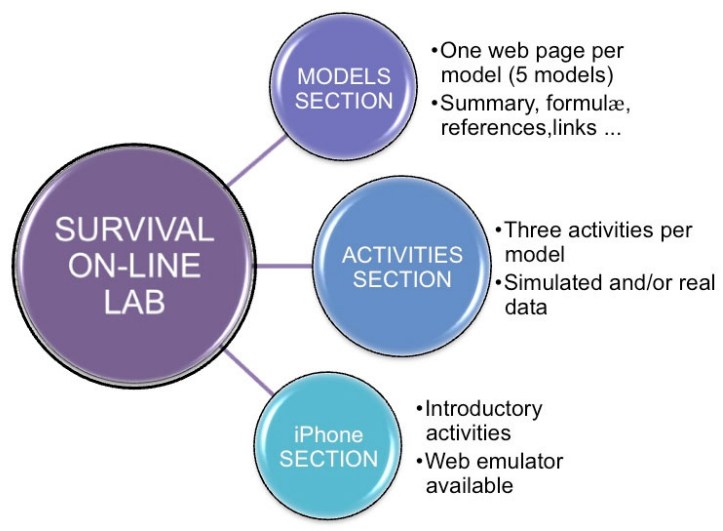

Figure 1. Organization of the Survival on-line lab

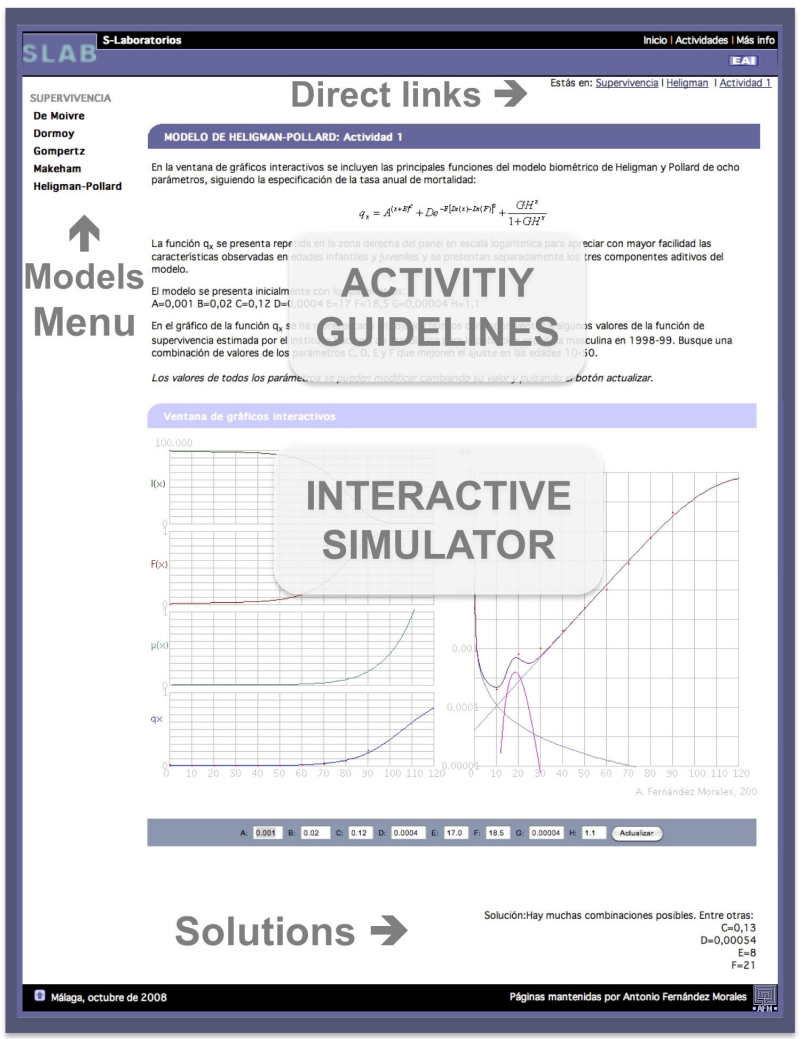

Figure 2. An activity web page of the on-line Survival lab 
The interactive graphics window of the simulator is displayed on the bottom part of the screen. The parameter values can be modified by the students, who get in real time the induced reaction of all the functions in the model. The simulator is then the main tool to solve all the problems and questions included in the activities. The tasks involved in the solution of the activities are varied, and may demand the specification of a new model with a better adjustment to real data, getting some additional information from the model, or even proposing an alternative specification that verifies some restrictions.

As an attempt to promote a more autonomous learning process, the solutions of the tasks of every activity are included at the bottom right end of the web page. Therefore, students can work with the simulator by themselves, individually or in groups. However, a link to the teacher's e-mail is also present to help them with the most difficult cases if needed.

The new iPhone/iPod touch section has been designed to provide students with a new learning tool that allows a greater mobility in their learning experience. The lite version of the simulator has been specifically developed for the iPhone or iPod touch screen (Fig. 3) and has a more limited number of features. The mobile version of the survival lab only includes three short activities related to the problem of the 'accident hump' observed in may countries in the mortality of young males. These three activities are deliberately simple in order to be carried out in a short time to overcome some of the main disadvantages of mobile devices in mobile learning, conditioned by the small screen size [4]. Furthermore, they constitute a good introductory stage before using the more complex web-based simulator. For this reason, the activities in the iPhone section have been programmed in a first stage, followed by the ones in the main section of the Survival laboratory.

For the students who have not an iPhone or an iPod touch, an html-based emulation of the iPhone simulator activities has been developed, which is available on-line. Thus, there are not access restrictions for this m-learning resource in our course.

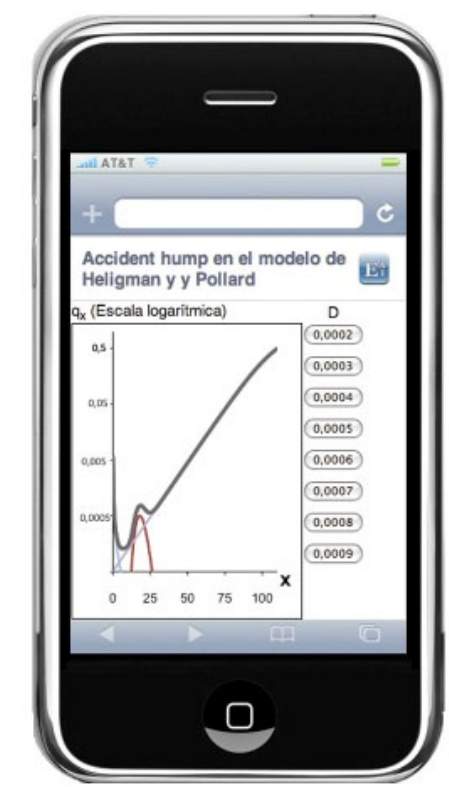

Figure 3. The lite version of the simulator on the iPhone

\section{B. The simulator}

The graphical simulator used for the on-line survival lab activities was developed as a series of Java applets embedded in the corresponding web pages. There are five versions of the applet, according to the most well known analytical survival models in the Actuarial field and their specific properties.

Having in mind that the potential users of the simulator are first semester students, the applet has been designed with the intention of providing an easy-to-use learning tool, with a very simple interface, and clearly focused on the main functions of the survival model and its fit to real observed data. Moreover, these considerations also contribute to a better effectiveness over the learning process, allowing the students to focus on the concepts rather on only paying attention to the technology or the software [3].

The simulator's interface has two panels (see Fig. 2). On the upper side, the graphical panel shows the functions of the survival model that describes human mortality, the number of survivors in a cohort, $1_{x}$, the distribution function of the random variable X: "age at death", $\mathrm{F}(\mathrm{x})$, the instant force of mortality, $\mu_{\mathrm{x}}$, and the probability of dying within 1 year for a person aged $\mathrm{x}$ exactly, $\mathrm{q}_{\mathrm{x}}$.

The applet for the Heligman-Pollard model also shows a plot of the function $\mathrm{q}_{\mathrm{x}}$ in log-scale on the right side. In this additional plot, the function $\mathrm{q}_{\mathrm{x}}$ is decomposed into three components that represent infant, accident and adult mortality: This decomposition constitutes the main feature of the Heligman-Pollard model.

The lower part contains the control panel. This panel displays the parameter values and a button to update in real time the graphical panel. Students can modify any of the parameters values and then refresh the graphical panel to observe the reaction of the model's functions.

Depending on the model, the number and composition of the parameters varies, ranging from one parameter for the simplest model (Dormoy) to eight for the HeligmanPollard model. Currently, the models incorporated are the following:

- Dormoy model

- De Moivre model

- Gompertz model

- Makeham model (first Makham law)

- Heligman-Pollard model (first Heligman-Pollard law)

For some activities, the graphical display shows red dots, corresponding to an observed data set of human mortality, in one of the functions of the model. The aim of these activities consists of simulating models and comparing them with observed mortality data, enhancing the scope of the designed learning activities.

A simplified version of the simulator has been developed for the iPhone/iPod platform, as mentioned before. This simpler version is designed by means of Javascript animations and only allows predetermined values for three (one at a time) of the eight parameters of the HeligmanPollard model. The student modifies the parameter's values clicking on the buttons on the right side of the screen (Fig. 3). 


\section{A PRELIMINARY ASSESSMENT}

A survey was carried out at the end of the last course to have a preliminary assessment of the efficiency and usefulness of the on-line survival lab for the learning process of our students. All the students participating in the experience answered a short questionnaire, which includes five items related to satisfaction with different aspects of the learning experience, three more associated to the usefulness of the carried out activities, and the last two related to their attitude towards more activities of this type in the course. Table 1 shows the main results obtained form the survey, distinguishing between the general activities section on the web and the iPhone specific one.

First, the results of the general activities section will be discussed. The best-valued items within the group of satisfaction are the clarity of the texts and questions (with a mean of 4.14 in a 1-5 scale) and the quality of the design and presentation (a mean of 4.08). The questions related to ease of use and ease of navigation obtained slightly lower means, (3.69 and 3.89). These results suggest that there is still work to do in improving the usability of the simulator and the web environment in which it is embedded.

The perceived level of difficulty of the activities has been valued on average with 2.53 (in a 1-5 scale), which is close to the center of the scale 1-5. However, there was a certain level of dispersion with an $8 \%$ students assigning the smallest value in the scale of difficulty (1) in contrast with a $14 \%$ who considered that the difficulty was considerable high (4 in the scale).

The results of the section of the questionnaire that includes the usefulness items are very positive. On one hand, students considered that the activities of the on-line survival lab are very useful for understanding survival models. This is confirmed with the very high mean of the answers to this question, 4.53. On the other hand, usefulness for autonomous learning and for general learning have also been valued with relatively high means $(3.92$ and 3.50 , respectively), especially the one concerning autonomous learning.

The last two questions in the survey were included to test our students' attitude towards more activities like the ones they did in the on-line lab. To this respect, it was found that the vast majority $(92 \%)$ would like more activities in the course program. As a consequence, for the next academic year it is very likely that the number of programmed activities based on the simulator will increase, with a more varied nature. In addition, even more students answered that they would do more activities with the simulator $(97 \%)$. This result is explained by the fact that the course in Actuarial Statistics I also includes an individual project, and many students thought it would be a good idea to use the simulator at some stage of their projects.

The results of the survey with reference to the iPhone/iPod touch activities are shown in the third column of Table I. Although the activities on this platform have an introductory nature and the simulator in this part of the online laboratory is a simplified version, there are not major differences in the results of the survey with respect to that of the general section. In general, the items related to quality, and ease of navigation and use are better valued for the iPhone section. Only the clarity of the texts and questions shows a smaller mean than in the case of the general section, which is likely due to the necessity of reducing the texts to a very short length to be displayed in the small screen of this mobile device.

In addition, the perceived usefulness for understanding analytical survival models and for autonomous and general learning is smaller than the observed in the general section of the on-line laboratory. These results were expected, considering that there are only three activities in the iPhone/iPod touch platform and are simpler than the cases in the general section. Lastly, the number of students demanding more activities in the iPhone platform is also smaller than the corresponding one for the web section.

TABLE I.

RESULTS OF THE SURVEY

\begin{tabular}{|l|c|c|}
\hline Questions & Web & iPhone \\
\hline Satisfaction with: (1-5 scale) & $\underline{\text { Mean }}$ & $\underline{\text { Mean }}$ \\
Difficulty level of the activities & 3.53 & 2.81 \\
Ease of use of the simulator & 4.14 & 4.11 \\
Clarity of the texts and questions & 3.89 & 4.76 \\
Ease of navigation & 4.08 & 4.24 \\
Quality of the design and presentation & $\underline{\text { Mean }}$ & $\underline{\text { Mean }}$ \\
\hline Usefulness for: (1-5 sacle) & 4.53 & 4.08 \\
Understanding survival models & 3.50 & 3.48 \\
General learning & 3.92 & 3.78 \\
Autonomous learning & $\underline{\text { Yes (\%) }}$ & $\underline{\text { Yes (\%) }}$ \\
\hline Attitude towards more activities (Yes/No) & - \\
Will you do more activities with the simulator? & 97 & 76 \\
Do you like more activities of this type? & 92 & 76 \\
\hline $\mathrm{n}=43$ & \\
\hline
\end{tabular}

\section{CONCLUSIONS}

Teaching and learning analytical survival models in the first course of the Degree in Actuarial Science often faces the problem of dealing with students who show little motivation and/or insufficient mathematical skills. This difficulty, that is frequently present in many Statistics courses in Higher Education, is even more serous in our case due to the central role that stochastic modeling of human survival plays in the whole Actuarial curriculum. As an attempt to overcome this problem, it is possible to design new learning strategies and tools based on the new technologies of communication and information that may incentive higher students' motivation and a better academic performance.

The on-line survival lab described in this paper, which integrates a series of activities based on an interactive graphical simulator of survival models, was designed to reduce, at least partially, the difficulties mentioned above. Students can concentrate in the interpretation of the models and datasets features, rather than in the most complicated mathematical calculations since the simulator obtains and draws automatically all the functions of each model. Consequently, students can get a deeper understanding of the different survival models characteristics and behavior.

Moreover, simulation based activities constitute a very interesting line of development in this field that has the potential of allowing the implementation in web pages through tools like Java applets. A great advantage of using the simulator implemented as a Java applet is the combination of remote access with interactivity and the possibility of experimentation. While the former increases the flexibility of the learning process and stimulates the auto 
organization of the students' time, the latter is a key element to incentive motivation.

Besides, the addition of a lite version of the simulator on the iPhone/iPod touch platform with three associated introductory activities has increased the variety on the nature of the activities and served as a first stimulus to enter in the more complex simulator of the on-line survival laboratory.

Finally, the results of the survey carried out at the end of the last course and the academic performance results are very positive. The students involved in the project perceived a high degree of usefulness of the experience, especially for a better understanding of survival models (the main objective of the on-line survival laboratory) and also for improving their skills related to autonomous learning. Another indicator of the good results of this experience is the high percentage of students demanding more activities of this kind in the course program.

\section{REFERENCES}

[1] C.M. Anderson-Cook and S. Dorai-Raj, "A Active Learning InClass Demonstration of Good Experimental Design," Journal of Statistics Education, vol. 9, no.1, 2001.

[2] J. Byrne, C. Heavey and P.J. Byrne, "A review of Web-based simulation and supporting tools," Simulation Modelling Practice and Theory, 18, pp. 253-276, 2010. doi:10.1016/j.simpat. $\underline{2009.09 .013}$

[3] B. Chance, D. Ben-Zvi, J. Garfield and E. Medina, "The Role of Technology in Improving Student Learning of Statistics", Technology Innovations in Statisitcs Education, Vol. 1, no. 1, 2007.

[4] W. Daher, "Students' Perceptions of Learning Mahematics with Cellular Phones and Applets", International Journal of Emerging Technologies in Learning, vol. 4, no. 1, March 2009.

[5] R.C. delMas and J. Garfield, "A Model of Classroom Research in Active Developing Simulation Activities to Improve Students' Statistical Reasoning," Journal of Statistics Education, vol. 7, no. 3, 1999 .

[6] Dikovic, L. "Implementing Dynamic Mathematics Resources with GeoGebra at the College Level", International Journal of Emerging Technologies in Learning, vol. 4, no.3, September 2009.

[7] I.D. Dinov, N. Christou and J. Sanchez, J. "Central Limit Theorem. New SOCR Applet and Demonstration Activity," Journal of Statistics Education, vol. 16, no. 2, 2008.
[8] A. Fernández-Morales, "Un Laboratorio Web Basado en Java para la Enseñanza de la Concentración Estacional en Series de Turismo," Estudios Turísticos, no. 146, 2000.

[9] A. Fernández-Morales, "Teaching Seasonality with SISMO: An On-Line Interactive Seasonal Simulator," in Educational Technology, Vol. III, A. Méndez-Vilas, J.A. Mesa-González and I. Sólo de Zaldívar, Eds. Consejería de Educación, Ciencia y Tecnología, Junta de Extremadura, Badajoz, 2002.

[10] P. Forster, "Assessing Technology Based approaches for Teaching and Learning Mathematics," International Journal of Mathematical Education in Science and Technology, no.37, 2006, pp. 145164.

[11] A. Littlejohn, I. Falconer and L. Mcgill, "Characterising effective eLearning resources", Computers \& Education, 50, pp. 757-771, 2008. doi:10.1016/j.compedu.2006.08.004

[12] M. Lunsford, G Holmes-Rowell and T. Goodson-Espy, T. "Classroom Research: Assessment of Student Understanding of Sampling Distributions of Means and the Central Limit Theorem in Post-Calculus Probability and Statistics Classes," Journal of Statistics Education, 14, 2006.

[13] T. Monahan, G. McArdle and M. Bertolotto, "mCLEV-R. Design and Evaluation of an Interactive and Collaborative M-Learning Application", International Journal of Emerging Technologies in Learning, vol. 2, no. 7, 2007.

[14] K. Schneiter, "Two Applets for Teaching Hypothesis Testing", Journal of Statistics Education, vol. 16, no. 3, 2008.

[15] J. Symanzik and N. Vukasinovik, "Teaching an Introductory Statistics Course with CyberStats, an Electronic Textbook," Journal of Statistics Education, 14, 2006.

[16] R.W. West and R.T. Ogden, "Interactive Demonstrations for Statistics Education on the World Wide Web," Journal of Statistics Education, vol. 6, no.3, 1998.

\section{AUTHORS}

A. Fernández-Morales is with the Department of Applied Economics (Statistics and Econometrics, Department 68), University of Málaga, 29071 Málaga, Spain (email: afdez@uma.es).

This work was supported in part by the Servicio de Innovación Educativa $y$ Servicio de Enseñanza Virtual y Laboratorios Tecnológicos of the University of Málaga (Project PIE08-025 'Adquisición y evaluación de competencias en un marco de aprendizaje contextualizado y participativo').

Manuscript received 20 July 2010. Published as resubmitted by the authors March $1^{\text {st }}, 2011$. 